\title{
Positive Psychology Intervention: A Focus Group Discussion
}

\author{
Darabi Mitra* \\ University of Aberdeen, Aberdeen, United Kingdom
}

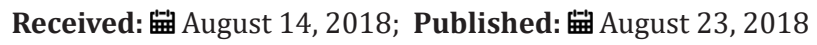

*Corresponding author: Darabi, Mitra, University of Aberdeen, Aberdeen, United Kingdom

\section{Opinion}

The content analysis of the focus group discussion to evaluate a positive psychology intervention (the three good things exercise) identified four principal themes:

a. Theme1: Identifying positives when things are hectic indicates that the three good things exercise helped academics to find positives in a day when their work did not go as well as expected. Therefore, the positive psychology intervention was useful and practical in assisting participants to find positives no matter how small or big in terms of importance and this made them happier.

b. Theme 2: The positive consequences of the three good things represents how this exercise changed academics' awareness/ perception of the events. Initially it was easy for them to write about their negative experiences of a day, but the exercise helped them to stop and think about how to find positive things in their day. Seligman, Rashid and Peterson (2005) found that the three good things exercise can help people to finish their day by shifting from the negative aspect of event to a positive and enjoyable memory of the event and that seems to have occurred with this group.

c. Theme 3: The negative aspects of practicing the three good things specifies some problems that academics experienced during the five day positive psychology intervention. For example finding a third good thing has been identified as a hard part of this task for all academics. The intervention was quite new for all the participants and it was only a five-day exercise; it can therefore be argued that, if the length of the intervention was longer they could possibly cope better and improved at doing it. Academics also found that identifying the reasons behind good events was problematic. It could be hard to attribute the good things to internal or external sources. These difficulties might be related to cultural differences as the three good things exercise is very American with its focus on positivity, very representative of a culture that tells you, "Have a nice day" all the time and that this enforced positivity might seem a little strange to British audience as indeed it did. Some of the participants found it difficult to identify more than one positive thing and mentioned that it was not what they normally did. However, the group did adjust to the demands of the exercise quite quickly suggesting that it was not a big issue.

d. Theme 4: Persistence of the exercise revealed that some academics are devoted to continue the exercise in the future as it helped them to think positively and made them aware that there are some positive things in their daily lives that needed to be identified. Similarly, Sheldon and Lyubomirsky (2007) found participants' self-reports of continued practice of positive activities after the intervention period can predict sustained increases in positive affect at a two week follow-up (Layous \& Lyubomirsky, 2011). Conversely some academics thought it was a good exercise for them but they preferred to do when they need it, for example when they feel stressed, not as a regular exercise. Research suggests that increasing positive emotions during a stressful time is regarded as an adaptive coping strategy (Folkman \& Moskowitz, 2000, 2004; cited in Layous \& Lyubomirsky, 2011). The participants were not asked to continue their exercise after the post assessment. It is therefore not clear whether any of them continued the exercise between post-assessment and the two week follow-up. However, as the sample size was quite small, it is not significant whether they continue their exercise or not. 
(C) (1) This work is licensed under Creative

To Submit Your Article Click Here:

Submit Article

DOI: 10.32474/LOJNHC.2018.01.000121

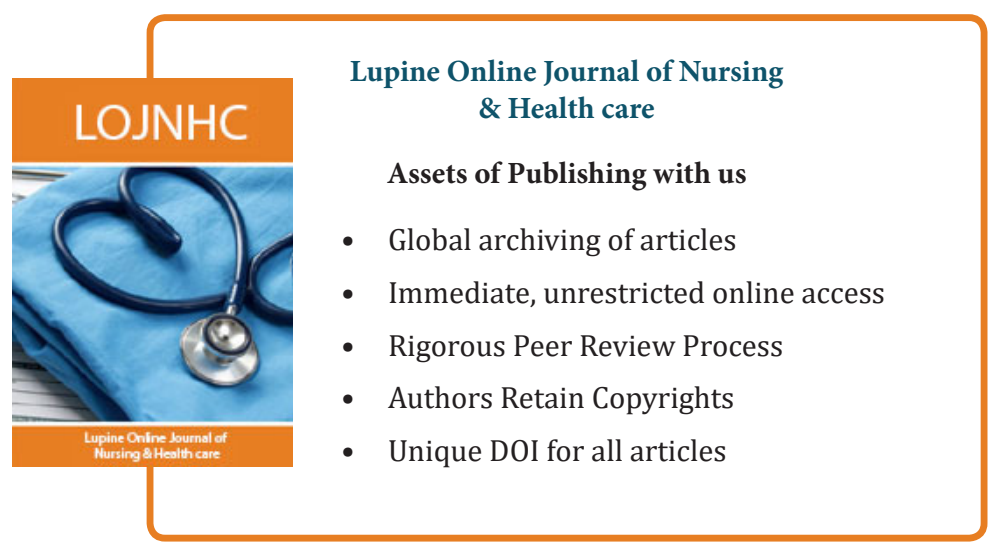

